\title{
Kinetic analysis of morphological differentiation and protease production in Streptomyces albidoflavus SMF301
}

\author{
Sung Gyun Kang and Kye Joon Lee \\ Author for correspondence: Kye Joon Lee. Tel: +82 28806705 . Fax: + 822882 9285/888 4911. \\ e-mail:1kj12345@plaza.snu.ac.kr
}

Department of Microbiology and Research Centre for Molecular Microbiology, Seoul National University, Seoul 151-742, Korea

\begin{abstract}
The effects of specific growth rate and specific nutrient uptake rate on morphological differentiation of Streptomyces albidoflavus SMF301 were determined in chemostat cultures. Production of three types of proteases: chymotrypsin-like protease (CTP), trypsin-like protease (TLP) and metalloprotease (MTP) were analysed in relation to mycelium growth and spore formation. Production of CTP was closely linked to mycelium growth, whereas spore formation, TLP synthesis and MTP synthesis were inversely related to growth. Evaluation of various kinetic parameters [specific production rates of spores $\left(q_{\text {spo }}\right), \operatorname{TLP}\left(q_{T P P}\right), \operatorname{MTP}\left(q_{M T P}\right)$ and $\left.\operatorname{CTP}\left(q_{C T P}\right)\right]$ showed that mycelium growth rate and CTP production were optimal at $0.1 \mathrm{~h}^{-1}$, but submerged spore formation, TLP production and MTP production were optimal at $0.025 \mathrm{~h}^{-1}$. Changes in specific nutrient uptake rates [glucose $\left(q_{\text {glu }}\right)$, ammonium ion $\left(q_{\mathrm{amn}}\right)$ and phosphate $\left.\left(\boldsymbol{q}_{\mathrm{pho}}\right)\right]$ affected sporulation and protease production; limitation of carbon, nitrogen and phosphate stimulated spore, TLP and MTP production.
\end{abstract}

Keywords: Streptomyces albidoflavus SMF301, submerged spore, kinetics of sporulation, extracellular proteases, differentiation

\section{INTRODUCTION}

Physiological differentiation, such as secondary metabolite production and morphological differentiation, in Streptomyces spp. may be induced by limitation of essential nutrients (Chater, 1984; Champness, 1988; Champness et al., 1989). The effects of essential nutrients on differentiation have been reported: limitation of nitrogen and phosphate can initiate sporulation (Ensign, 1988; Glazebrook et al., 1990), but the effect of glucose addition differs in various species of Streptomyces (Babcock \& Kendrick, 1988; Chater et al., 1988; Daza et al., 1989).

Morphological differentiation of Streptomyces coelicolor is accompanied by the production of degradation enzymes (Chater, 1989; Granozzi et al., 1990). Various extracellular proteases participate in the assimilation of external proteinaceous nitrogen sources (Shapiro, 1989) and a recent study of the regulation of protease activities during growth and mycelium development of Streptomyces exfoliatus SMF13 showed that the production of

Abbreviations: CTP, chymotrypsin-like protease; MTP, metalloprotease; TLP, trypsin-like protease. trypsin-like protease (TLP) and leupeptin-inactivating protein are closely related to morphological differentiation (Kim \& Lee, 1995, 1996).

The effects of nutrient limitation on morphological and physiological differentiation have been determined mostly in agar cultures where the concentration of nutrients and products change significantly with time. Under these conditions, the roles of specific nutrients or products cannot be distinguished. Although only a minority of Streptomyces form spores in submerged liquid cultures (Kendrick \& Ensign, 1983; Daza et al., 1989; Koepsel \& Ensign, 1984; Glazebrook et al., 1990), such cultures provide advantages over agar cultures for determining the specific role of environmental changes on differentiation.

We have investigated a strain of Streptomyces albidoflavus SMF301 that produces extracellular proteases and abundant spores in both submerged and agar cultures (Rho et al., 1992; Shin \& Lee, 1986). The cellular composition of submerged spores differs from those of mycelium and aerial spores (Lee \& Rho, 1993). Kinetic analysis showed that the specific submerged spore formation rate $\left(q_{\mathrm{spo}}\right)$ is inversely related to the specific mycelium growth rate $(\mu)$. In the batch cultures, limi- 
(a)

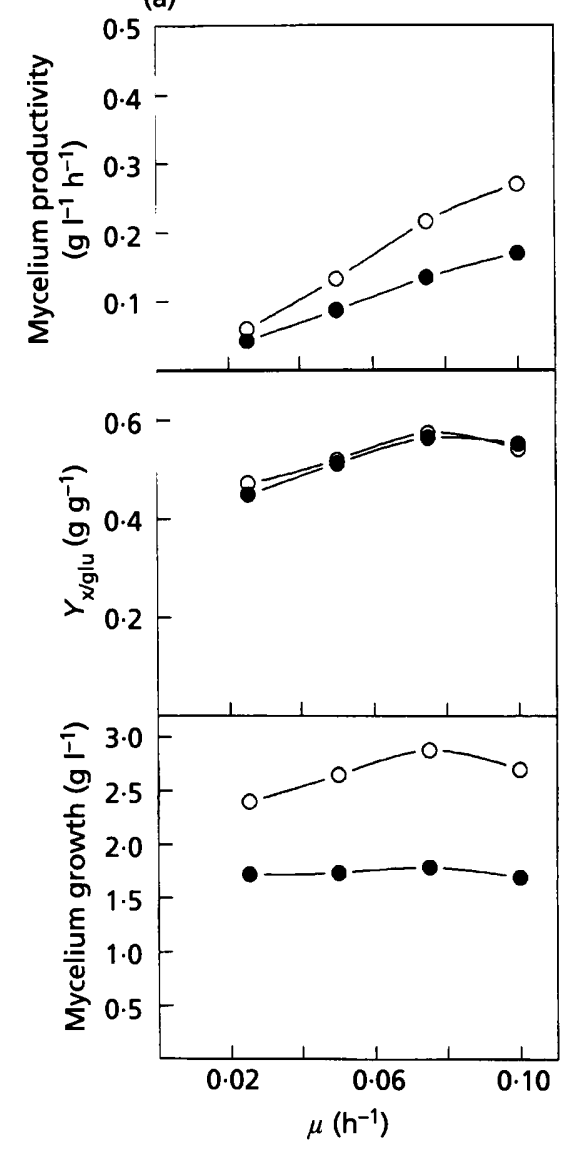

(b)

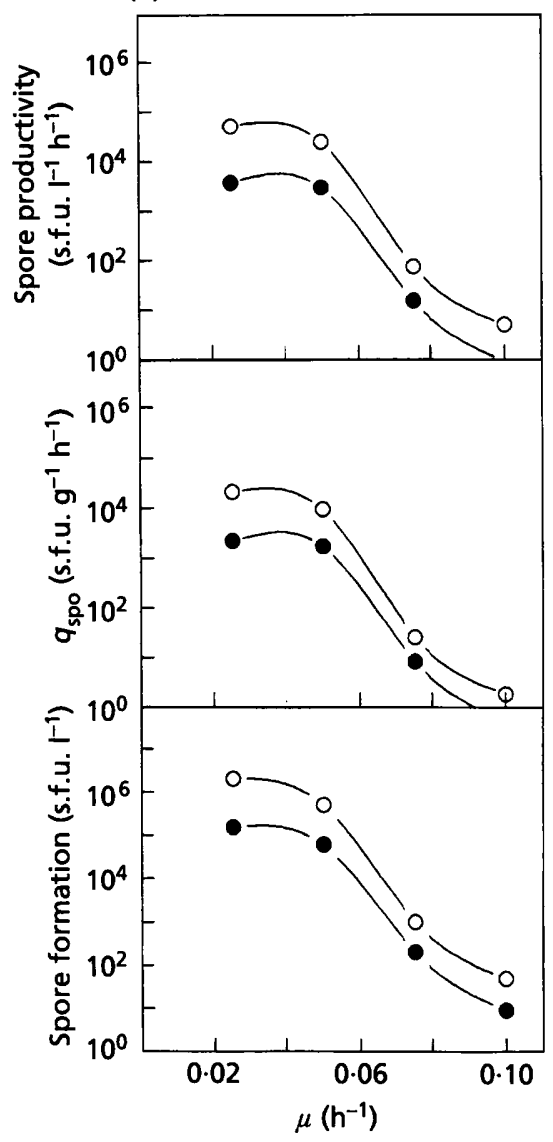

(c)

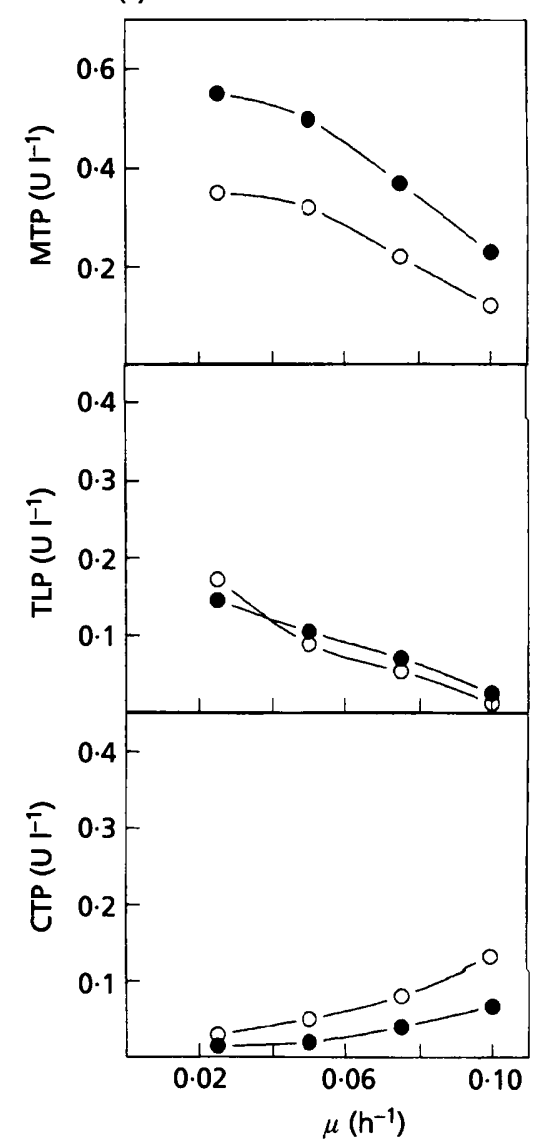

Fig. 1. Effect of $\mu$ on (a) mycelium growth, $Y_{x / g l u}$ and mycelium productivity; (b) spore formation, $q_{\text {spo }}$ and spore productivity (s.f.u., spore-forming units); and (c) changes in CTP, TLP and MTP in S. albidoflavus SMF301. O, Carbonlimited culture;, , nitrogen-limited culture.

tation of nitrogen and phosphate appears to stimulate sporulation (Rho \& Lee, 1994). Extracellular proteases were identified in the culture supernatant; the three major types of proteases, chymotrypsin-like protease (CTP), metalloprotease (MTP) and TLP, showed association with growth and spore formation in submerged culture (Kang et al., 1995a, b).

In this study we analysed quantitatively the effects of specific growth rate and specific nutrient uptake rate on mycelium growth, spore formation and extracellular protease production in chemostat cultures of $S$. albidoflavus SMF301.

\section{METHODS}

Organism and media. Streptomyces albidoflavus SMF301 has been described by Rho et al. (1992). Stock agar culture medium consisted of $(\mathrm{w} / \mathrm{v}): 1 \%$ glucose, $0.2 \%$ peptone, $0.3 \%$ yeast extract, $0 \cdot 1 \%$ beef extract and $1 \cdot 8 \%$ agar. The carbonlimited medium for the chemostat consisted of $(\mathrm{w} / \mathrm{v}) ; 0.5 \%$ glucose, $0.2 \% \mathrm{NH}_{4} \mathrm{Cl}, 0.01 \% \mathrm{KH}_{2} \mathrm{PO}_{4}, 0.03 \% \mathrm{MgSO}_{4} .7 \mathrm{H}_{2} \mathrm{O}$, $0.001 \% \quad \mathrm{FeSO}_{4} .7 \mathrm{H}_{2} \mathrm{O}, 0.001 \% \quad \mathrm{CuSO}_{4} .5 \mathrm{H}_{2} \mathrm{O}, \quad 0.001 \%$ $\mathrm{CaCl}_{2} \cdot 2 \mathrm{H}_{2} \mathrm{O}$ and $0.0003 \% \mathrm{MnCl}_{2} \cdot 4 \mathrm{H}_{2} \mathrm{O}$. In the nitrogenlimited cultures, $1 \%$ glucose and $0.05 \% \mathrm{NH}_{4} \mathrm{Cl}$ were used as carbon and nitrogen sources, respectively. In the phosphatelimited cultures, $1 \%$ glucose, $0.2 \% \quad \mathrm{NH}_{4} \mathrm{Cl}$ and $0.01 \%$ $\mathrm{KH}_{2} \mathrm{PO}_{4}$ were used as carbon, nitrogen and phosphate sources, respectively. The initial $\mathrm{pH}$ of the media was adjusted to $7 \cdot 0$ before steam sterilization. Phosphate and other salts were separately sterilized by membrane filtration $(0.2 \mu \mathrm{m}$, Millipore).

Strain maintenance and culture conditions. Strains were maintained by transfer to slopes of stock culture medium each month, and were stored at $4{ }^{\circ} \mathrm{C}$. For agar cultures, a spore suspension harvested from the stock culture was spread evenly and incubated at $28^{\circ} \mathrm{C}$. For submerged batch cultures, one loopful of mycelium and spores was used to inoculate $30 \mathrm{ml}$ of the seed culture medium and incubated at $28^{\circ} \mathrm{C}$ for $2 \mathrm{~d}$. The seed culture was used to inoculate 1.3 I of the chemostat culture medium contained in a jar fermenter (Model KF-5L, Korea Fermentor Co.). Cultures were maintained at $28^{\circ} \mathrm{C}$ and the $\mathrm{pH}$ was controlled at $7 \cdot 0$ with agitation at 200 r.p.m. and aeration at $1 \mathrm{v} \mathrm{v}^{-1} \mathrm{~min}^{-1}$.

Observation of mycelium morphology and growth. Morphological characteristics were observed with a phase-contrast microscope (Nikon Laphot). To measure growth, mycelium was harvested by centrifugation $(10000 \mathrm{~g}, 10 \mathrm{~min})$ and washed twice with physiological saline solution and once with distilled water. The washed mycelium was collected by vacuum 
filtration (Whatman GF/C paper), dried at $80^{\circ} \mathrm{C}$ for $24 \mathrm{~h}$ and weighed.

Analysis of chemical changes in the culture medium. The concentration of glucose was determined with dinitrosalicylic acid reagent (Miller, 1959). The concentration of ammonium ion was measured with a specific ion analyser (Model EA940, Orion Research). The phosphate concentration was determined by the method of Pierpoint (1957).

Measurement of submerged spore formation. The culture broth $(5 \mathrm{ml})$ was sonicated for $5 \mathrm{~min}$ at $100 \mathrm{~W}$ using a sonic dismembrator (Model 300, Fisher). The sonicated suspension $(0.5 \mathrm{ml})$ was mixed gently with $0.1 \mathrm{M} \mathrm{HCl}(4.5 \mathrm{ml})$ and after $5 \mathrm{~min}$ the mixture was diluted in physiological saline and spread on stock agar medium. Colonies were counted after $4 \mathrm{~d}$ incubation to estimate the number of spores in the inoculum (Lee \& Rho, 1993).

Assay of proteases. The culture broth was centrifuged at $(10000 \mathrm{~g}, 10 \mathrm{~min})$ and enzyme activities were measured in the supernatant. Total protease activity was estimated by measuring tyrosine liberated from Hammarsten casein (Merck) during $15 \mathrm{~min}$ incubation at $37^{\circ} \mathrm{C}$ and $\mathrm{pH} 7.5(0.1 \mathrm{M}$ Tris/ $\mathrm{HCl}$ buffer). One unit of casein hydrolytic activity (caseinase) was defined as the amount of enzyme needed to produce $1 \mu \mathrm{g}$ tyrosine equivalent $\mathrm{min}^{-1}$ from the casein (Narahashi, 1970). Other hydrolytic activities were estimated by measuring the amount of $p$-nitroanilines liberated from the following synthetic substrates: $N$-benzoyltyrosine $p$ nitroanilide (BTPNA; a specific substrate for CTP), $N$ benzoylarginine $p$-nitroanilide (BAPNA; a specific substrate for TLP) and leucyl $p$-nitroanilide (LPNA; a specific substrate for MTP).

Solutions containing $200 \mu \mathrm{M}$ aminoacyl $p$-nitroanilides were incubated at $35^{\circ} \mathrm{C}$ and $\mathrm{pH} 7.5(0.1 \mathrm{M}$ Tris $/ \mathrm{HCl}$ buffer $)$. Activity was calculated from the linear part of the curve using $\varepsilon_{405}=9620 \mathrm{~mol}^{-1} \mathrm{~cm}^{-1}$. One unit of hydrolytic activity was defined as the amount of enzyme needed to produce $1 \mu \mathrm{mol}$ p-nitroaniline $\min ^{-1}$ (Sarath et al., 1989).

Gel electrophoresis. Protease activity was visualized by incorporating $0.1 \%$ gelatin into a SDS-PAGE gel $(12 \%)$. After electrophoresis at $4{ }^{\circ} \mathrm{C}$, the gel was incubated at $35^{\circ} \mathrm{C}$ for $3 \mathrm{~h}$ in $0 \cdot 1 \mathrm{M}$ potassium phosphate buffer $(\mathrm{pH} 7 \cdot 5)$ and then stained with Coomassie brilliant blue.

Fermentation kinetic parameters. Data from chemostat cultures were analysed for specific growth rate $(\mu)$, specific ammonium ion uptake rate $\left(q_{\mathrm{amn}}\right)$, specific glucose uptake rate $\left(q_{\mathrm{glu}}\right)$, specific phosphate uptake rate $\left(q_{\mathrm{pho}}\right)$, specific rate of spore formation $\left(q_{\mathrm{spo}}\right)$ and specific rate of protease production $\left(q_{\mathrm{TLP}}, q_{\mathrm{MTP}}\right.$ and $\left.q_{\mathrm{CTP}}\right)$. Kinetic parameters in chemostat cultures were calculated as follows: $\mu=D$, where $D$ is the dilution rate $\left(\mathrm{h}^{-1}\right) ; q_{\mathrm{glu}}=D\left(s_{\mathrm{o}}-\bar{s}\right) / \bar{x}$, where $s_{\mathrm{o}}$ is the concentration of glucose in the medium, $\bar{s}$ is the steady-state concentration of glucose $\left(\mathrm{g}^{-1}\right)$ and $\bar{x}$ is the steady-state concentration of biomass $\left(\mathrm{g} \mathrm{l}^{-1}\right) ; q_{\mathrm{amn}}=D\left(n_{\mathrm{o}}-\bar{n}\right) / \bar{x}$, where $n_{\mathrm{o}}$ is the concentration of ammonium ions in the medium and $\bar{n}$ is the steady-state concentration of ammonium ions $\left(\mathrm{g} \mathrm{l}^{-1}\right) ; q_{\mathrm{pho}}=$ $D\left(p_{0}-\bar{p}\right) / \bar{x}$, where $p_{0}$ is the concentration of phosphate in the medium and $\bar{p}$ is the steady-state concentration of phosphate ions $\left(\mathrm{g} \mathrm{l}^{-1}\right) ; q_{\mathrm{spo}}=D(\overline{s p o}) / \bar{x}$, where $\overline{s p o}$ is the steady-state number of spores; $q_{\mathrm{TLP}}=D(\overline{T L P}) / \bar{x}$, where $\overline{T L P}$ is the steadystate TLP activity; $q_{\mathrm{MTP}}=D(\overline{M T P}) / \bar{x}$, where $\overline{M T P}$ is the steady-state MTP activity; $q_{\mathrm{CTP}}=D(\overline{C T P}) / \bar{x}$, where $\overline{C T P}$ is the steady-state CTP activity; and $Y_{\mathrm{spo} / \mathrm{x}}=s p o / x, \mathrm{Y}_{\mathrm{x} / \mathrm{glu}}=$ $\bar{x} /\left(s_{0}-\bar{s}\right)$ and $Y_{\mathrm{x} / \mathrm{amn}}=\bar{x} /\left(n_{0}-\bar{n}\right)$, as suggested by Pirt (1975), where $Y$ is the maximum growth yield.

\section{RESULTS}

\section{Kinetic analyses of growth, spore formation and protease production}

In chemostats limited by glucose or ammonium ion, the steady-state values for biomass concentration increased with $\mu$ (Fig. 1a); on the other hand, the number of submerged spores in the chemostats was inversely related to $D$ (Fig. 1b). Kinetic parameters calculated from the steady-state values showed that $Y_{\mathrm{x} / \mathrm{glu}}$ was optimal at ca $0 \cdot 1 \mathrm{~h}^{-1}$, while $q_{\text {spo }}$ was optimal at ca $0.025 \mathrm{~h}^{-1}$ (Fig. 1b). $q_{\text {glu }}$ and $q_{\mathrm{amn}}$ increased with increasing $\mu$ (Fig. 1), indicating that those nutrients were essential for mycelium growth but not for spore formation.

Patterns of extracellular protease production were

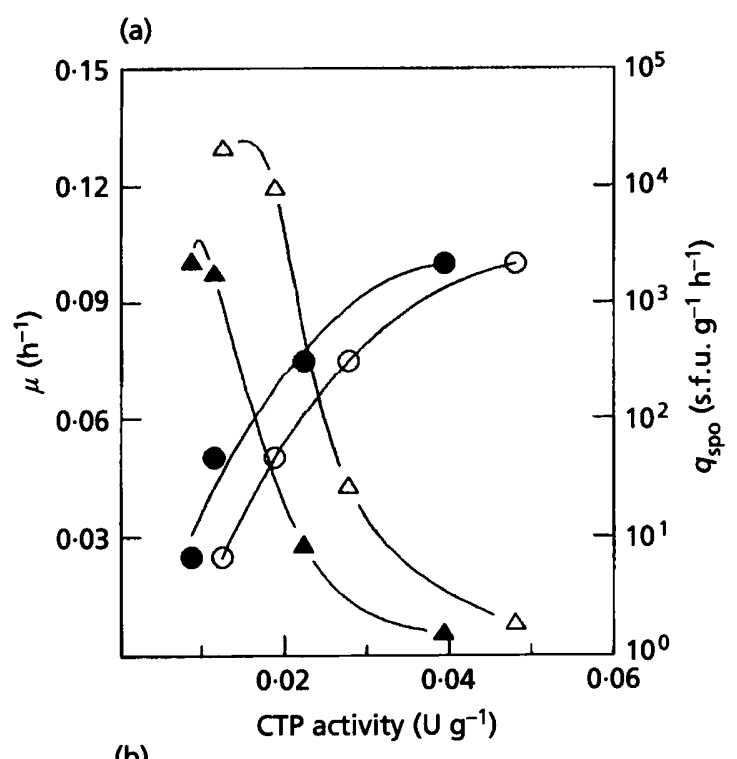

(b)

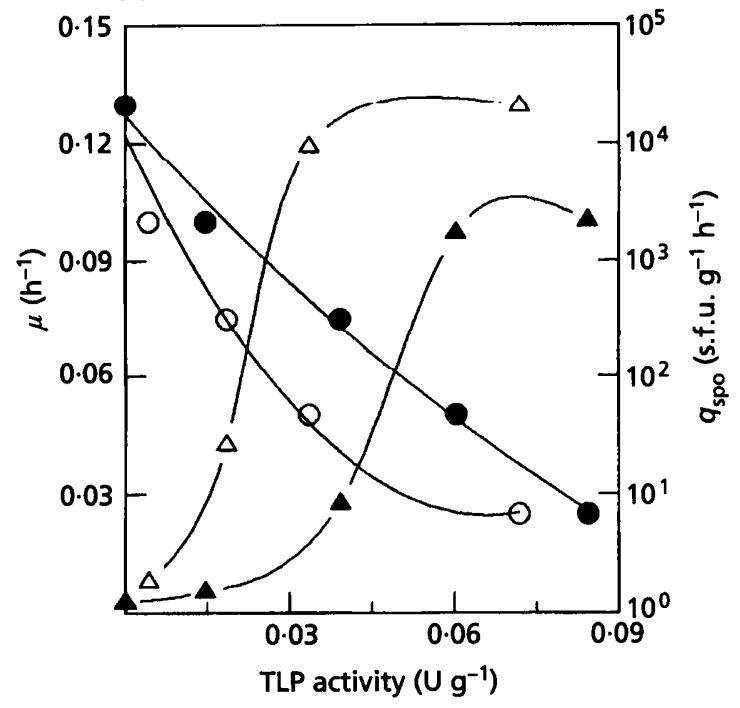

Fig. 2. Relationship between (a) CTP or (b) TLP activity and changes in $\mu(0, O)$ and $q_{\text {spo }}(\Delta, \Delta)$. O, $\Delta$, Carbon-limited culture;, $\mathbf{\Delta}$, nitrogen-limited culture. 
(a)

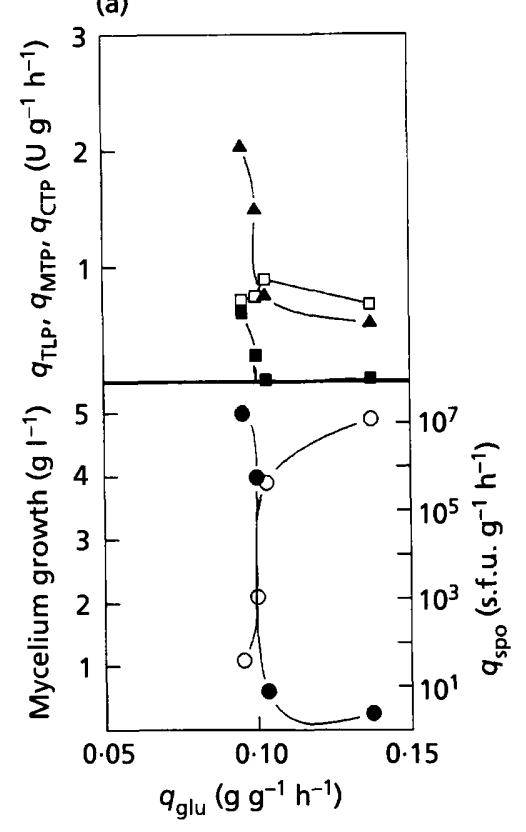

(b)

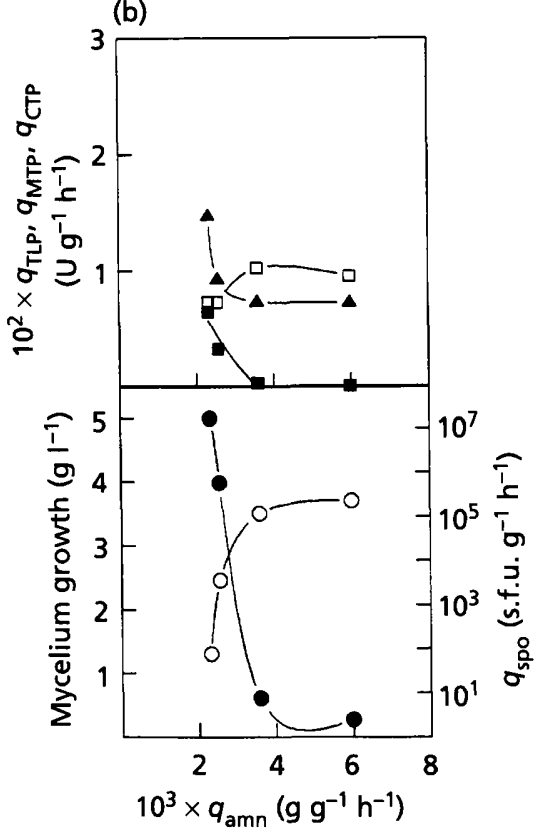

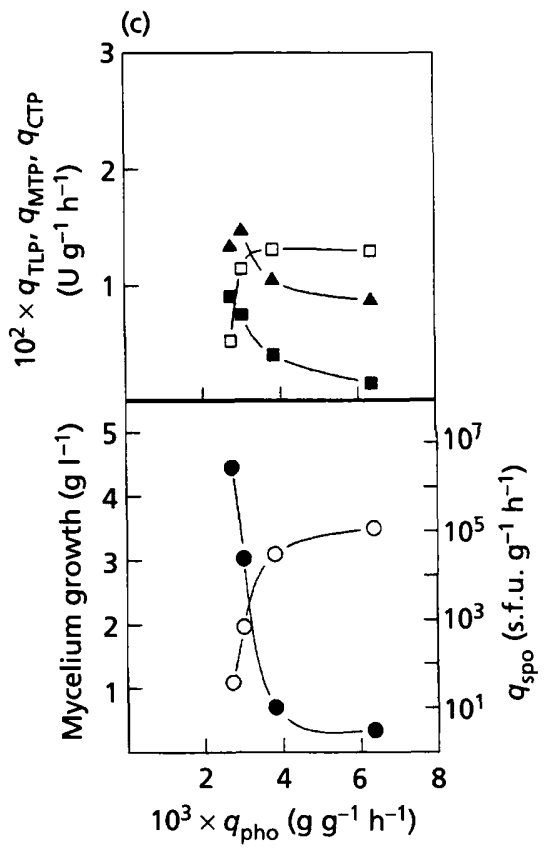

Fig. 3. (a) Effect of $q_{\text {glu }}$ on mycelium growth $(0), q_{\mathrm{spo}}(\boldsymbol{Q}), q_{\mathrm{TLP}}(\boldsymbol{\square}), q_{\mathrm{MTP}}(\boldsymbol{\Delta})$ and $q_{\mathrm{CTP}}(\square)$. (b) Effect of $q_{\mathrm{amn}}$ on mycelium growth (O), $q_{\text {spo }}(\boldsymbol{O}), q_{\mathrm{TLP}}(\boldsymbol{\square}), q_{\mathrm{MTP}}(\boldsymbol{\Delta})$ and $q_{\mathrm{CTP}}(\square)$. (c) Effect of $q_{\text {pho }}$ on mycelium growth $(O), q_{\mathrm{spo}}(\mathbf{Q}), q_{\mathrm{TLP}}(\boldsymbol{\square}), q_{\mathrm{MTP}}$ (A) and $q_{C T P}(\square)$. $D$ was fixed at $0.06 \mathrm{~h}^{-1}$ and $\mathrm{pH}$ was controlled at 7 with $1 \mathrm{M} \mathrm{HCl}$ and $1 \mathrm{M} \mathrm{NaOH}$.

determined by activity staining (data not shown); the three major types of proteases, CTP, TLP and MTP were detected. As shown in Fig. 1(c), the activity of CTP was directly related to $\mu$, whereas TLP activity and MTP activity were inversely related to $\mu$. Fig. 2 shows more clearly the relationships between enzyme activity and the morphological characteristics of cells. The results indicate that CTP was correlated with mycelium growth, and that TLP and MTP were associated with spore formation.

\section{Effects of nutrient uptake rate on growth, spore formation and protease production at a fixed growth rate}

Although spore formation and TLP production were enhanced at the low growth rates of both carbon- and nitrogen-limited cultures, it was not clear whether the nutrients played a role in the differentiation of mycelium and protease production. To distinguish the effect of the growth rate from the effects of the nutrient limitation, chemostat cultures were carried out at a fixed $\mu$ of $0.06 \mathrm{~h}^{-1}$ where the concentration of glucose, ammonium ion or phosphate was varied by changing the feed concentration.

In the chemostat where the glucose feed rate was varied, the changes in growth and $q_{\text {CTP }}$ were directly related to $q_{\mathrm{glu}}$. In contrast, $q_{\mathrm{spo}}, q_{\mathrm{MTP}}$ and $q_{\mathrm{TLP}}$ were inversely related to $q_{\mathrm{glu}}$ (Fig. 3a). Similar patterns were found in chemostats where the ammonium ion or phosphate feed rate was varied (Fig. 3 b and c).

\section{Effect of nutrient balance on growth, spore formation and protease production at a fixed growth rate}

In chemostat cultures at a fixed $\mu$ of $0.06 \mathrm{~h}^{-1}$ in chemically defined media with different $\mathrm{C} / \mathrm{N}$ ratios, $q_{\mathrm{spo}}$ was affected by the change in the $\mathrm{C} / \mathrm{N}$ ratio, and the changes in $q_{\mathrm{TLP}}$ and $q_{\mathrm{MTP}}$ were correlated with the change in $q_{\text {spo }}$ (Fig. 4). The analysis of results with varied $\mathrm{C} / \mathrm{N}$ ratios also shows that the production of spores, TLP and MTP was very high at both low and high $\mathrm{C} / \mathrm{N}$ ratios. The results from changing nutrient feed rates and $\mathrm{C} / \mathrm{N}$ ratios indicate that limitation of any one of the essential nutrients (carbon and nitrogen) brings about differentiation. On the other hand, a change in $q_{\text {CTP }}$ was associated with mycelium growth change rather than spore formation.

\section{DISCUSSION}

As reported previously (Kang et al., 1995a), morphological differentiation of $S$. albidoflavus SMF301 is accompanied by sequential production of CTP, TLP and MTP. Each protease was thought to have a specific role in growth and differentiation, CTP production being linked to mycelium formation, whereas TLP and MTP were associated with spore formation. In chemostats, the kinetic analyses of changes in protease and spore formation can be carried out with variation in only one parameter such as growth rate or supply of an essential nutrient.

The specific rate of CTP production and the mycelium 


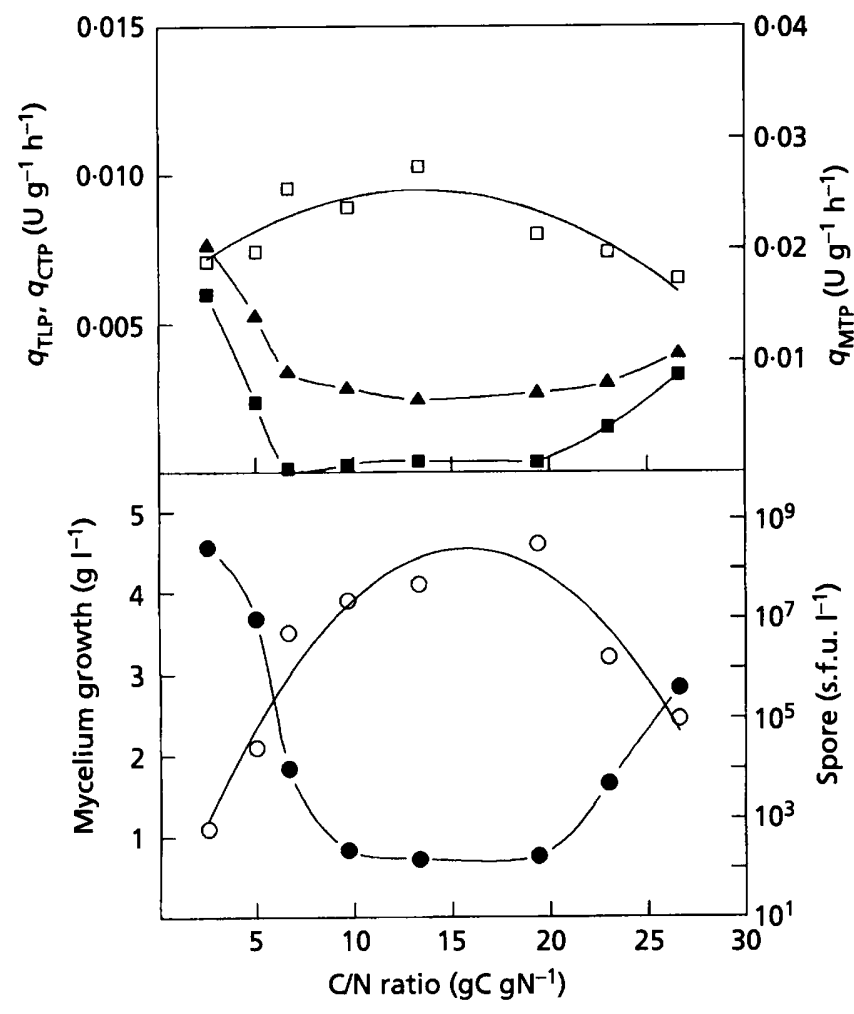

Fig. 4. Effect of $C / N$ ratio in the nutrient input on the steadystate values in chemostat cultures $\left(D, 0.06 \mathrm{~h}^{-1}\right)$. 0 , mycelium growth;, spore; $\square, q_{\mathrm{TLP}} ; \Delta, q_{\mathrm{MTP}} ; \square, q_{\mathrm{CTP}}$.

growth rate responded similarly to changes in growth rate and nutrient feed rates. In addition, the activity of CTP in various chemically defined media was much lower than that in rich media containing sodium caseinate as sole nitrogen source (Kang et al., 1995a; Shin \& Lee, 1986). This suggests that CTP could be a major protease supporting mycelium growth in complex media by hydrolysis of proteinaceous compounds.

On the other hand, previous reports (Kang et al., 1995a, b) demonstrated that the activities of TLP and MTP were not affected by medium composition. The kinetic analyses also showed that the specific production rates of TLP and MTP were associated with the changes of in the spore production rate observed under various conditions. These results suggest that TLP and MTP are closely correlated with spore formation.

Limitation of essential nutrients is reported to have a variety of effects on differentiation in Streptomyces. Sporulation of several species was not inhibited by high concentrations of glucose (Daza et al., 1989) and the repression of sporulation by glucose has been attributed to acid accumulation and $\mathrm{pH}$ (Babcock \& Kendrick, 1988; Rho \& Lee, 1994). However, in our experiment (Fig. 3), where only the carbon feed rate was changed, kinetic analyses show that the rate of spore formation was clearly inhibited by high concentrations of glucose and stimulated by low concentrations. Since the $\mathrm{pH}$ of all chemostats was maintained at 7 , the inhibition was not caused by acid accumulation or $\mathrm{pH}$ variation. These results are consistent with the observation that cellular differentiation in surface cultures was inhibited by high concentrations of glucose (Chater et al., 1988).

Submerged spore formation is initiated by depletion of ammonium ions or phosphate in Streptomyces griseus (Kendrick \& Ensign, 1983; Rho \& Lee, 1994) and is suppressed by excess nitrogen source (Glazebrook et al., 1990; Rho \& Lee, 1994). The kinetic data reported here show that limitation of carbon, nitrogen or phosphate brings about differentiation. It is also noteworthy that nutrient imbalances in the $\mathrm{C} / \mathrm{N}$ ratio (high $\mathrm{C} / \mathrm{N}$, nitrogen limitation; low $\mathrm{C} / \mathrm{N}$, carbon limitation) were detrimental to mycelium proliferation but beneficial to spore formation, TLP and MTP synthesis (Fig. 4).

Thus, it was concluded that morphological differentiation in S. albidoflavus is triggered by limitation of any one of the essential nutrients (glucose, nitrogen or phosphate). In addition, nutrient limitation immediately affected the production of proteases that play important physiological roles in mycelium growth and spore formation: CTP participated in mycelium growth, and MTP and TLP were associated with the formation of spores.

\section{ACKNOWLEDGEMENTS}

This work was supported by a research grant from the Research Centre for Molecular Microbiology (RCMM) sponsored by the Korea Science and Engineering Foundation (KOSEF). The authors are very grateful to Professor K. F. Chater for his thoughtful discussion.

\section{REFERENCES}

Babcock, M. J. \& Kendrick, K. E. (1988). Cloning of DNA involved in sporulation of Streptomyces griseus. J Bacteriol 170, 2802-2808.

Champness, W. C. (1988). New loci required for Streptomyces coelicolor morphological and physiological differentiation. $J$ Bacteriol 170, 1168-1174.

Champness, W. C., Adamidis, T. \& Riggle, P. E. (1989). Developmental genes and antibiotic biosynthesis. In Genetics and Molecular Biology of Industrial Microorganisms, pp. 108-112. Edited by C. L. Hershberger, S. W. Queener \& G. Hegeman. Washington, DC: American Society for Microbiology.

Chater, K. F. (1984). Morphological and physiological differentiation in Streptomyces. In Microbial Development, pp. 89-115. Edited by R. Losick \& L. Shapiro. Cold Spring Harbor, NY: Cold Spring Harbor Laboratory.

Chater, K. F. (1989). Sporulation in Streptomyces. In Regulation of Prokaryotic Development, pp. 277-299. Edited by I. Smith, R. A. Slepecky \& P. Setlow. Washington, DC: American Society for Microbiology.

Chater, K. F., Lawlor, E. J., Mendez, C., Bruton, C. J., Davis, N. K., Plaskitt, K., Guthrie, E. P., Daly, B. L., Baylis, H. A. \& Trong, K. V. (1988). Gene expression during Streptomyces development. In Biology of Actinomycetes '88. Proceedings of the 7th International Symposium for Biology of Actinomycetes, pp. 64-70. Edited by Y. Okami. Tokyo: Japan Scientific Societies Press. 
Daza, A., Martín, J. F., Dominguez, A. \& Gil, J. A. (1989). Sporulation of several species of Streptomyces in submerged culture after nutritional downshift. J Gen Microbiol 135, 2483-2491.

Ensign, J. C. (1988). Physiological regulation of sporulation of Streptomyces griseus. In Biology of Actinomycetes '88. Proceedings of the 7th International Symposium for Biology of Actinomycetes, pp. 309-315. Edited by Y. Okami. Tokyo: Japan Scientific Societies Press.

Glazebrook, M. A., Doull, J. L., Stuttard, C. \& Vining, L. C. (1990). Sporulation of Streptomyces venezuelae in submerged cultures. J Gen Microbiol 136, 581-588.

Granozzi, C., Billeta, R., Passantino, R., Sollazzo, M. \& Puglia, A. M. (1990). A breakdown in macromolecular synthesis preceding differentiation in Streptomyces coelicolor A3(2). J Gen Microbiol 136, 713-716.

Kang, S. G., Kim, I. S., Rho, Y. T. \& Lee, K. J. (1995a). Production dynamics of extracellular proteases accompanying morphological differentiation of Streptomyces albidoflavus SMF301. Microbiology 141, 3095-3103.

Kang, S. G., Kim, I. S., Ryu, J. G., Rho, Y. T. \& Lee, K. J. (1995b). Purification and characterization of trypsin like protease and metalloprotease in Streptomyces albidoflavus SMF301. J Microbiol 33, 307-314.

Kendrick, K. E. \& Ensign, J. C. (1983). Sporulation of Streptomyces griseus in submerged culture. J Bacteriol 155, 357-366.

Kim, I. S. \& Lee, K. J. (1995). Physiological roles of leupeptin and extracellular proteases in mycelium development of Streptomyces exfoliatus SMF13. Microbiology 141, 1017-1025.

Kim, I. S. \& Lee, K. J. (1996). Trypsin-like protease of Streptomyces exfoliatus SMF13, a potential agent in mycelial differentiation. Microbiology 142, 1797-1806.
Koepsel, R. \& Ensign, J. C. (1984). Microcycle sporulation of Streptomyces viridochromogenes. Arch Microbiol 140, 9-14.

Miller, G. L. (1959). Use of dinitrosalicylic acid reagent for determination of reducing sugar. Anal Chem 31, 426-428.

Lee, K. J. \& Rho, Y. T. (1993). Characteristics of spores formed by surface and submerged cultures of Streptomyces albidoflavus SMF301. J Gen Microbiol 139, 3131-3137.

Narahashi, Y. (1970). Pronase. Methods Enzymol 19, 651-664.

Pierpoint, W. S. (1957). The phosphatase and metaphosphatase activities of pea extract. Biochem J 65, 67-76.

Pirt, S. J. (1975). Principle of microbe and cell cultivation. Oxford: Blackwell.

Rho, Y. T., Kim, H.T., Oh, K. H., Kang, H. I., Ward, A. C., Goodfellow, M., Hah, Y.C. \& Lee, K. J. (1992). Numerical identification of a Streptomyces strain producing spores in submerged culture. Korean J Microbiol 30, 278-285.

Rho, Y. T. \& Lee, K. J. (1994). Kinetic studies on mycelium growth and spore formation in submerged cultures of Streptomyces albidoflavus SMF301. J Gen Microbiol 140, 2061-2065.

Sarath, G., De La Motte, R. S. \& Wagner, F. W. (1989). Protease assay methods. In Proteolytic Enzymes: A Practical Approach, pp. 25-55. Edited by R. J. Beynone \& J. S. Bond. Oxford, New York \& Tokyo: IRL Press.

Shapiro, S. (1989). Nitrogen assimilation in actinomycetes and the influence of nitrogen nutrition on actinomycete secondary metabolism. In Regulation of Secondary Metabolism in Actinomycetes, pp. 149-153. Edited by S. Shapiro. Boca Raton, FL: CRC Press.

Shin, H. S. \& Lee, K. J. (1986). Regulation of extracellular alkaline protease biosynthesis in a strain of Streptomyces spp. Korean J Microbiol 24, 32-37.

Received 31 December 1996; revised 30 April 1997; accepted 7 May 1997. 